\title{
History matters: childhood weight trajectories as a basis for planning community-based obesity prevention to adolescents
}

\author{
Joakim Ekberg, Marianne Angbratt, L. Valter, M. Nordvall and Toomas Timpka
}

\section{Linköping University Post Print}

N.B.: When citing this work, cite the original article.

Original Publication:

Joakim Ekberg, Marianne Angbratt, L. Valter, M. Nordvall and Toomas Timpka, History matters: childhood weight trajectories as a basis for planning community-based obesity prevention to adolescents, 2012, International Journal of Obesity, (36), 4, 524-528.

http://dx.doi.org/10.1038/ijo.2011.263

Copyright: Nature Publishing Group http://npg.nature.com/

Postprint available at: Linköping University Electronic Press

http://urn.kb.se/resolve?urn=urn:nbn:se:liu:diva-77329 
ORIGINAL ARTICLE

History matters: childhood weight trajectories as a basis for planning community-based obesity prevention to adolescents Joakim Ekberg MSc ${ }^{1}$, Marianne Angbratt $\mathrm{PhLic}^{2}$, Lars Valter $\mathrm{PhLic}^{2}$, Maria Nordvall MD, $\mathrm{PhD}^{3,4}$, Toomas Timpka MD, $\mathrm{PhD}^{1,2}$

${ }^{1}$ Section of Community Medicine, Department of Medical and Health Sciences, Linköping University, Linköping, Sweden

${ }^{2}$ Centre for Public Health, Östergötland County Council, Linköping, Sweden

${ }^{3}$ Division of Pediatrics, Department of Clinical and Experimental Medicine, Faculty of Health Sciences, Linköping University, Linköping, Sweden

${ }^{4}$ Vrinnevi Hospital, Norrköping, Sweden

Correspondence: Joakim Ekberg, Department of Medical and Health Sciences, Faculty of Health Sciences, Linköping University, S-581 83 Linköping, Sweden. Tel.: +46 101032380 ; fax: +46 10 1031865. E-mail: joakim.ekberg@liu.se Conflict of interest statement: None

Running head: Weight history matters 


\section{Abstract}

Objective: To use epidemiological data and a standardized economic model to compare projected costs for obesity prevention in late adolescence accrued using a cross-sectional weight classification for selecting adolescents at age 15 years compared with a longitudinal classification.

Methods: All children born in a Swedish county (population 440 000) in 1991 who participated in all regular measurements of height and weight at ages 5, 10, and 15 years $(\mathrm{n}=4312)$ were included in the study. The selection strategies were compared by calculating the projected financial load resulting from supply of obesity prevention services from providers at all levels in the health care system. The difference in marginal cost per 1000 children was used as the primary end point for the analyses.

Results: Using the cross-sectional selection strategy, 3.8\% of adolescents at age 15 years were selected for evaluation by a paediatric specialist, and $96.2 \%$ were chosen for population-based interventions. In the trajectory-based strategy, $2.4 \%$ of the adolescents were selected for intensive paediatric care, $1.4 \%$ for individual clinical interventions in primary health care, $14.0 \%$ for individual primary obesity prevention using the Internet, and $82.1 \%$ for population-based interventions. Costs for the cross-sectional selection strategy were projected to USD463 581 per 1000 adolescents and for the trajectory-based strategy were USD 302016 per 1000 adolescents.

Conclusions: Using projections from epidemiological data, we found that by basing the selection of adolescents for obesity prevention on weight trajectories, the load on highly specialized paediatric care can be reduced by one third and total health service costs for obesity management among adolescents reduced by one third. Before use in policies and prevention program planning, our findings warrant confirmation in prospective costbenefit studies. 


\section{Introduction}

In many countries, obesity prevention programs are delivered during childhood by wellchild health centres to preschool children and thereafter by school health care throughout compulsory school. However, there are few countries where support for reaching or maintaining a healthy weight is provided to youths graduating from compulsory school. When taking on this challenge, it is vital to bear in mind that any local population of adolescents aged 15 years consists of individuals with different genetic predispositions, e.g. for homeostatic weight regulation. ${ }^{1}$ The adolescents have also lived in social and physical environments that differ with regard to how these predispositions are expressed. ${ }^{2}$ When choosing appropriate interventions, an isolated measurement of body mass index (BMI) at age 15 years may thus disregard important information. Evidence has been accumulated on the effectiveness of interventions for obesity prevention and therapy at individual and population levels. At the individual level, a recent Cochrane Database Systematic Review ${ }^{3}$ summarized $^{-}$ efficacy data on interventions that can be supplied in late adolescence. For obese individuals, pharmaceutical treatments in combination with lifestyle intervention for 6 months were concluded to be the most effective. Dietary interventions were the only non-pharmaceutical intervention shown to be effective, but usually require long-term treatment and strict adherence. At the population level, studies have shown that Internet-based intervention programs have led to positive changes in diet and exercise habits among young people, ${ }^{4}$ but also pointed at a low number of users and low compliance. ${ }^{5,6}$

Using longitudinal BMI data into adulthood categories as reference, age-specific childhood BMIs have been identified for classifying children into normal weight, overweight, and obesity categories. ${ }^{7}$ These categories have been reported to have high specificity but low sensitivity for predicting obesity and metabolic disorders in young adulthood. ${ }^{8}$ This study sets out to use epidemiological data and a standardized economic model to compare projected 
costs for obesity prevention in late adolescence accrued using a cross-sectional weight classification for selecting adolescents at age 15 years compared with a longitudinal classification. The difference between the classifications in projected marginal cost per 1000 adolescents is used as the primary end point for the analyses. Because disturbance to the homeostatic systems regulating body weight is hard to correct, ${ }^{2}$ a structured record of changes in weight classifications may provide more specific evidence for planning of obesity interventions directed at adolescents when graduating from compulsory school. Weight and height data from a total cohort of children born during 1 year in a Swedish county was used for the analyses.

\section{Weight trajectory mechanisms}

In children and in adults, the homeostasis of weight is regulated by feedback mechanisms controlled by the central nervous system. In lean individuals, insulin ${ }^{9}$ and leptin ${ }^{10}$ are the important hormonal mediators. ${ }^{11}$ In obese individuals, however, insulin and leptin have significantly less importance. Recent research has shown that energy homeostasis is controlled by several closely integrated systems, implying that the efficacy of interventions targeting one neuronal subset or signal transduction pathway is limited by compensatory

responses elsewhere. ${ }^{11}$ There is agreement that during caloric restriction, neuronal input from hormones activate responses that favour food intake. On the other hand, it is unclear whether the reverse is true, that is, whether or not adiposity negative feedback at caloric load protects against increased weight gain. Until this controversy is settled, central nervous system resistance to insulin and leptin in obesity cannot be used as the sole biologic model in the design of obesity prevention programs and therapeutic interventions on already obese patients. Alternative explanations, for example, that obesity among children is the result of an inherent lack of protection against environmental factors, ${ }^{12}$ should still be taken into consideration. Nonetheless, present evidence favours the interpretation that disturbances to 
the system regulating energy homeostasis are hard to correct, and that the trajectory of such disturbances has importance for future regulation.

\section{Materials and methods}

The study was performed in three steps. Weight and height data were collected at ages 5 , 10 and 15 years from all children born in 1991 in a Swedish county (population 440 000). Thereafter, categorization of children at age 15 years into cross-sectional weight classes and longitudinal weight trajectory groups was performed. The cross-sectional and trajectory-based selection strategies for different interventions were finally compared using the difference in the projected service costs as the end point measure.

\section{Data collection}

All children born in 1991 in the study county who had participated in all regular measurements of weight and height at ages 5, 10, and 15 years were included in the study ( $n=4312$ ). Informed consent was obtained from parents and children older than 12 years before data collection. Data on weight and height at age 5 years were collected from wellchild health centre records. Data on weight and height at ages 10 and 15 years were gathered from records at the school health service in 2002 and 2007. For administrative reasons, schools in the county recorded weight and height at different semesters, which influenced the exact measurement age (Table 1). Reasons for missing data included incomplete health records, children moving out of the county in their first 15 years, individuals declining to participate, individuals with physical or mental disorders excluded by choice of their schools, and individuals with protected identity. The final data set with complete data at all three measurement points included 4312 individuals (2059 girls and 2253 boys). 


\section{Selection strategies}

The cross-sectional selection strategy was based on the organizational routines in the study county at the time of the final data collection (2006-2007). The paediatric clinics offered medical evaluation to obese children graduating from compulsory school at age 15 years. The clinics had no formal guidelines for provision of care to these children following the evaluation, but options ranged from pharmaceutical to surgical procedures. Surgery was normally considered for patients with BMI $>35 \mathrm{~kg} / \mathrm{m}^{2}$. Overweight and normal weight children not evaluated at paediatric clinics were provided population-based interventions consisting of information campaigns from the Public Health Centre at the County Council.

The alternative trajectory-based selection strategy was combined with projected weighthistory sensitive interventions at four levels according to the estimated risk for persistent obesity. At level I, children with a history of constant obesity were to be referred for intensive paediatric care including pharmaceutical and/or surgical interventions. Level II provides individual-based interventions administered in primary care to individuals who have become obese in adolescence. A medical assessment that excludes underlying clinical conditions is provided, followed by a long-term behavioural-dietary intervention. At level III, overweight adolescents previously of normal weight were to be informed using the Internet about the health risks associated with being overweight (motivational wake-up). Overweight adolescents with a history of overweight are provided access to a periodic check-up and support using the Internet (motivational check-up). Overweight children previously obese who have lost considerable weight are given assistance to sustain their efforts using the Internet (motivational support). At level IV, population-based primary obesity prevention was supposed to be supplied using the Internet by public health agencies to the entire remaining population of adolescents. 


\section{Data analysis}

The children in the study cohort were classified as normal weight, overweight or obese according to the International Obesity Taskforce (IOTF) criteria ${ }^{7}$ at each measurement age. For the cross-sectional strategy, the children were classified into one of three IOTF groups according to their BMI at 15 years. Interventions according to the practice routines in 20062007 were thereafter allocated to each group. For the trajectory-based strategy, the children were classified into one of 27 trajectory groups according to their history of weight classifications, and each trajectory was allocated to a program level. The decision tree for the strategy was then pruned using two heuristic rules: (1) obesity established at age 10 years indicates the presence of causative factors from childhood justifying immediate intensive care at age 15 years; and (2) the type of behavioural intervention against overweight at age 15 years is determined by the direction of the most recent weight change (increase, decrease or none from age 10 years). Following application of these rules, the weight classification at age 5 years was found not to influence the allocation of individuals to program levels. The weight classification histories could therefore be collapsed into nine trajectories (based on combination of weight classification at age 10 and 15 years).

The cross-sectional selection strategy was compared with the trajectory-based strategy by calculating the relative financial load resulting from supply of obesity prevention services to adolescents from providers at all levels in the health care system. Treatment costs per child for childhood obesity interventions by paediatric specialists and in primary care based on 2001 prices reported by Cater et al. ${ }^{13}$ (family-based program in primary care for obese children, USD1896; Orlistat therapy, USD1935; adjustable gastric binding, USD 31 553) were used for the analyses. Among patients referred to intensive paediatric care, it was estimated that gastric binding surgery was suggested for one third, pharmacological treatment for one third, and family-based program treatment for one third. Expenses for one 1-h 
individual Internet intervention per year were included in the trajectory program costs. Both selection strategies were allocated costs (USD10 per child) for population-based public health interventions.

\section{Results}

\section{Cross-sectional and trajectory-based classifications}

At age 15 years, the study cohort comprised $82.3 \%$ normal weight adolescents, $13.8 \%$ overweight individuals and $3.8 \%$ obese adolescents. Of the nine weight trajectories, each trajectory had at least seven children represented in the 1991 cohort (Table 2). $74.9 \%$ of the children were consistently classified as normal weight at age 10 and 15 years, $8.3 \%$ of the children were classified as overweight at age 10 and 15 years, and $2.4 \%$ of the children were consistently classified as obese at age 10 and 15 years. $4.1 \%$ of the children moved from being normal weight to overweight during the period and $1.4 \%$ moved from being non-obese to being obese.

\section{Comparison of selection strategies}

Using the cross-sectional selection strategy, $96.2 \%$ of adolescents at age 15 years were projected to be provided population-based obesity interventions, and 3.8\% were projected to be eligible for evaluation by a paediatric specialist. The total annual cost for the program based on the selection strategy was estimated to be USD463 581 per 1000 adolescents (Table 3).

In the trajectory-based program, $82.1 \%$ of adolescents at age 15 years were projected to be provided with population-based obesity interventions, and an additional $15.4 \%$ of the adolescents were projected to be provided with individual interventions using the Internet $(14.0 \%)$ or in primary care $(1.4 \%) .2 .4 \%$ of the adolescents would have been eligible for intensive paediatric care. Weight trajectories for each group in the trajectory-based 
intervention program for the adolescents are shown in Figure 1. The total annual cost of the program based on the selection strategy was estimated to be USD302 016 per 1000 adolescents.

Present weight

Figure 1 Weight trajectory-based selection of adolescents to obesity prevention intervention at age 15 years.

\section{Discussion}

Point estimates of childhood obesity using IOTF cut-off limits for BMI have been found to have low sensitivity (6-15\%) for predicting obesity and metabolic disorders in young adulthood. ${ }^{8}$ The present study was aimed at exploring weight trajectories for identification of adolescents at risk for developing obesity and metabolic disorders as adults, particularly children with a non-obese childhood BMI, and projecting the costs for a trajectory-adjusted strategy for obesity prevention. We used IOTF cut-off points to analyse the weight classification trajectories of 4312 children up to age 15 years. In addition to the obese children, the analysis selected non-obese children who had been overweight at both age 10 and 15 years ( $8.3 \%$ of the study population at age 15 years) and those who had become overweight during this time period ( $4.1 \%$ of the population) for individual preventive interventions. These results echo a recent study from the United States that analysed the 
weight trajectories of 3408 children. In this study, individuals who were overweight in early childhood and did not have a sustained decline in BMI after the age of 7 years and individuals who were not overweight in early childhood but had experienced annual increases in BMI were selected for obesity prevention in late adolescence. ${ }^{14}$ Similarly, an Australian study following 1197 children from birth to age 14 years described a "rising to moderate adiposity" trajectory with individuals who were never obese but nevertheless associated with high metabolic risk in late adolescence. ${ }^{15}$ That study recognized a larger "falling to moderate" trajectory $(22.5 \%)$ than our study (obese-overweight; $1.4 \%$ of the study population), but it did not recognize a stable overweight trajectory. Our obese-obese trajectory (2.4\% of the population) corresponds to the "stable high" obesity trajectory (including $7 \%$ of the population) described in the Australian study. ${ }^{15}$ The Australian "stable high" trajectory that was associated with markedly increased metabolic risk in late adolescence seems thus to include our obese-obese trajectory and overlap with the overweight-overweight trajectory. How such trajectory groups should be defined can be disputed. However, childhood obesity determined by the IOTF reference BMI cut-off points have been found to have a high specificity (97-99\%) for predicting obesity and metabolic disorders in young adulthood. ${ }^{8}$ That is, only a very small percentage of the individuals who are categorized in the low-risk groups in adulthood were obese as children. There are therefore reasons for categorizing children determined obese by the IOTF reference BMI cut-offs separately and providing them with extra resources for treatment and prevention. Moreover, our recent observations and those by others suggest that children belonging to the "constant overweight" ( $8.3 \%$ of the population at age 15 years in our study) and "turning overweight" ( $4.1 \%$ of the population) trajectories should also be considered as candidates for individual obesity prevention before they develop obesity in adulthood. 
The present need for obesity prevention among adolescents is greater than the resources available. Our projections indicate that the trajectory-based strategy would benefit adolescents with more specified interventions without increasing the costs. Online behavioural interventions open up the possibility of provide individuals at risk with obesity prevention programs. ${ }^{16}$ Promising approaches include Internet-based coaching programmes for high-risk patients. ${ }^{17}$ However, when interpreting our study, it must be taken into consideration that the results are limited in several aspects. Our economic projections were not calculated from costs recorded in the study county. Because the aim was to compare hypothetical intervention strategies, standardized cost data from the literature were used. Likewise, a marginal costs model assuming the presence of a health service infrastructure was used for the analysis. This model also included some hypothetical elements. For instance, in Sweden pharmaceutical treatment for obesity can only be supplied to patients less than 18 years of age after permission from the Medical Products Agency (MPA). The MPA can at short notice allow prescription of a specific drug to an individual patient by issuing a special license on a named patient basis. To establish the superiority of the trajectory strategy, it must be adapted, implemented, and evaluated within a health service context, and the factual costs recorded and analysed. Furthermore, we did not record a metabolic profile at age 15 years and have not collected BMI data in adulthood; the detailed metabolic risk for each trajectory group is thus not known for this population. We also contend that weight-related data may be lacking in school health care records at present and documentation from earlier interventions may be unavailable. However, with the wider use of electronic health records, the possibility of using weight trajectories to inform screening of adolescents when they leave school health care will increase.

Evidence on overweight and obesity based on weight history makes it possible to improve the performance of selection strategies when adolescents are provided with specific obesity 
preventive services. Using a projection from epidemiological data, we found that by basing the selection of adolescents for obesity prevention on weight trajectories, the load on highly specialized paediatric care can be reduced by one third and total health service costs for obesity management among adolescents reduced by one third. One out of five children can be selected for individualized interventions, such as health dialogue ${ }^{18}$ and motivational interviewing. ${ }^{19}$ Meanwhile, the load on highly specialized paediatric care, including surgical and pharmacological interventions, can be reduced and total health service costs for obesity interventions among adolescents can be reduced by one third. However, before use in policies and prevention program planning, the findings warrant confirmation in prospective costbenefit studies.

\section{Acknowledgments}

Annual ALF financing (a special government subsidy) was obtained from Östergötland County Council, Sweden. The funding agency did not influence the research process in any aspect. We are grateful to all school health care nurses and physicians who made this study possible.

\section{References}

1. Koeppen-Schomerus G, Wardle J, Plomin R. A genetic analysis of weight and overweight in 4-year-old twin pairs. Int J Obes Relat Metab Disord 2001; 25: 838844.

2. Han JC, Lawlor DA, Kimm SY. Childhood obesity. Lancet 2010; 375: 1737-1748.

3. Oude Luttikhuis H, Baur L, Jansen H, Shrewsbury VA, O'Malley C, Stolk RP, et al. Interventions for treating obesity in children. Cochrane Database Syst Rev 2009; (1): CD001872 (online). 
4. Thompson D, Baranowski T, Cullen K, Watson K, Liu Y, Canada A, et al. Food, fun, and fitness internet program for girls: pilot evaluation of an e-Health youth obesity prevention program examining predictors of obesity. Prev Med 2008; 47: 494-497.

5. Carter-Edwards L, Bastian LA, Schultz M, Amamoo MA, Ostbye T. An internetbased weight loss intervention initiated by a newspaper. Prev Chronic Dis 2009; 6: A101.

6. McConnon A, Kirk SF, Ransley JK. Process evaluation of an internet-based resource for weight control: use and views of an obese sample. J Nutr Educ Behav 2009; 41: 261-267.

7. Cole TJ, Bellizzi MC, Flegal KM, Dietz WH. Establishing a standard definition for child overweight and obesity worldwide: international survey. BMJ 2000; 320: 1240 1243.

8. Janssen I, Katzmarzyk PT, Srinivasan SR, Chen W, Malina RM, Bouchard C, et al. Utility of childhood BMI in the prediction of adulthood disease: comparison of national and international references. Obes Res 2005; 13: 1106-1115.

9. De Souza CT, Araujo EP, Bordin S, Ashimine R, Zollner RL, Boschero AC, et al. Consumption of a fat-rich diet activates a proinflammatory response and induces insulin resistance in the hypothalamus. Endocrinology 2005; 146: 4192-4199.

10. Munzberg H, Myers Jr MG. Molecular and anatomical determinants of central leptin resistance. Nat Neurosci 2005; 8: 566-570.

11. Morton GJ, Cummings DE, Baskin DG, Barsh GS, Schwartz MW. Central nervous system control of food intake and body weight. Nature 2006; 443: 289-295. 
12. Berthoud HR. Mind versus metabolism in the control of food intake and energy balance. Physiol Behav 2004; 81: 781-793.

13. Carter R, Moodie M, Markwick A, Magnus A, Vos T, Swinburn B, et al. Assessing cost-effectiveness in obesity (ACE-obesity): an overview of the ACE approach, economic methods and cost results. BMC Public Health 2009; 9: 419.

14. Reagan PB, Salsberry PJ. Pathways to adolescent overweight: body mass index and height percentile change in childhood. Int J Pediatr Obes 2010; 5: 80-87.

15. Huang R, de Klerk NH, Smith A, Kendall GE, Landau LI, Mori TA, et al. Lifecourse childhood adiposity trajectories associated with adolescent insulin resistance. Diabetes Care 2011; doi:10.2337/dc10-1809.

16. McCoy MR, Couch D, Duncan ND, Lynch GS. Evaluating an internet weight loss program for diabetes prevention. Health Promot Int 2005; 20: 221-228.

17. Goessens BM, Visseren FL, de Nooijer J, van den Borne HW, Algra A, Wierdsma J, et al. A pilot-study to identify the feasibility of an Internet-based coaching programme for changing the vascular risk profile of high-risk patients. Patent Educ Couns 2008; 73: $67-72$.

18. Lingfors H, Lindstrom K, Persson LG, Bengtsson C, Lissner L. Lifestyle changes after a health dialogue. Results from the Live for Life health promotion programme. Scand J Prim Health Care 2003; 21: 248-252.

19. Soderlund LL, Nordqvist C, Angbratt M, Nilsen P. Applying motivational interviewing to counselling overweight and obese children. Health Educ Res 2009; 24: 442-449. 
Table 1 Mean (SD) and median factual ages of the study cohort at the stipulated ages for data collection

\begin{tabular}{llllllllll}
\hline Data & \multicolumn{3}{l}{ Age $\sim 5$ years } & \multicolumn{3}{c}{ Age 10 years } & \multicolumn{5}{c}{ Age $~ 15$ years } \\
\cline { 2 - 10 } & All & Girls & Boys & All & Girls & Boys & All & Girls & Boys \\
\hline Range & $4.0-6.7$ & $4.1-6.7$ & $4.0-6.5$ & $8.8-11.7$ & $8.9-11.7$ & $8.8-11.7$ & $12.6-16.4$ & $12.6-16.4$ & $12.7-16.4$ \\
Median & 5.2 & 5.2 & 5.2 & 10.5 & 10.5 & 10.5 & 14.5 & 14.5 & 14.5 \\
Mean (SD) & $5.3(0.31)$ & $5.3(0.31)$ & $5.3(0.31)$ & $10.5(0.38)$ & $10.5(0.37)$ & $10.5(0.39)$ & $14.4(0.72)$ & $14.4(0.73)$ & $14.5(0.72)$ \\
$n$ & 4312 & 2059 & 2253 & 4312 & 2059 & 2253 & 4312 & 2059 & 2253 \\
\hline
\end{tabular}


Table 2 Weight trajectories according to IOTF BMI category at ages 10 and 15 years for children born in 1991 in Östergötland County $(n=4312)$ displayed by estimated risk levels for persistent obesity

\begin{tabular}{|c|c|c|c|c|}
\hline \multirow[t]{2}{*}{ Risk level } & \multicolumn{2}{|c|}{ Weight classification } & \multirow[b]{2}{*}{$n$} & \multirow[b]{2}{*}{ Percent } \\
\hline & Age $\sim 10$ years & Age $\sim 15$ years & & \\
\hline Level I & Obese & Obese & 104 & 2.4 \\
\hline \multirow[t]{2}{*}{ Level II } & Normal & Obese & 7 & 0.2 \\
\hline & Overweight & Obese & 55 & 1.3 \\
\hline \multirow[t]{4}{*}{ Level III } & Normal & Overweight & 179 & 4.2 \\
\hline & Overweight & Overweight & 357 & 8.3 \\
\hline & Obese & Overweight & 60 & 1.4 \\
\hline & Obese & Normal & 8 & 0.2 \\
\hline \multirow[t]{2}{*}{ Level IV } & Normal & Normal & 3230 & 74.9 \\
\hline & Overweight & Normal & 312 & 7.2 \\
\hline
\end{tabular}


Table 3 Projected annual costs for the trajectory-based and cross-sectional intervention programs

\begin{tabular}{|c|c|c|c|c|c|c|c|}
\hline \multicolumn{5}{|c|}{ Trajectory-based program } & \multicolumn{3}{|c|}{ Cross-sectional program } \\
\hline Risk level & $n$ & $\%$ & Cost per child (USD) & Total cost (USD) & $\begin{array}{l}\text { Risk } \\
\text { level }\end{array}$ & $\begin{array}{l}\text { Cost per } \\
\text { child (USD) }\end{array}$ & Total cost \\
\hline \multicolumn{5}{|l|}{ Level I } & \multicolumn{3}{|c|}{ Paediatric evaluation } \\
\hline & & & $1896(1 / 3)$ & 65728 & & $1896(1 / 3)$ & 65728 \\
\hline & 104 & 2.4 & $1935(1 / 3)$ & 67080 & & $1935(1 / 3)$ & 67080 \\
\hline & & & $31553(1 / 3)$ & 1093837 & & $31553(1 / 3)$ & 1093837 \\
\hline Total & 104 & 2.4 & & 1226645 & & & 1226645 \\
\hline \multicolumn{8}{|l|}{ Level II } \\
\hline & & & & & & $1896(1 / 3)$ & 39184 \\
\hline & 62 & 1.4 & 1896 & 117552 & & $1935(1 / 3)$ & 39990 \\
\hline & & & & & & $31533(1 / 3)$ & 651682 \\
\hline Total & 62 & 1.4 & & 11552 & & & 730856 \\
\hline \multicolumn{5}{|l|}{ Level III } & \multicolumn{3}{|c|}{ Population-based intervention } \\
\hline Wake-up & 179 & 4.2 & 35 & 6265 & & 10 & 1790 \\
\hline Check-up & 357 & 8.3 & 45 & 16065 & & 10 & 3570 \\
\hline Support & 60 & 1.4 & 35 & 6265 & & 10 & 600 \\
\hline Total & 596 & 13.8 & & 28595 & & & 5960 \\
\hline \multicolumn{8}{|l|}{ Level IV } \\
\hline Total & 3550 & 82.3 & 10 & 35500 & & 10 & 35500 \\
\hline $\begin{array}{l}\text { Total } \\
\text { program }\end{array}$ & 4312 & 100 & & 1302292 & & & 1998961 \\
\hline \multicolumn{4}{|c|}{ Cost per 1000 children } & 302016 & & & 463581 \\
\hline
\end{tabular}

